\title{
MLPA-conservation mechanism in wireless sensor network environments
}

\author{
Sheng-Tzong Cheng, Jia-Shing Shih", Tun-Yu Chang, Gwo-Jiun Horng and Chih-Lun Chou
}

\begin{abstract}
Transmission using excess power not only shortens the lifetime of sensor nodes, but also introduces interference. Packets should ideally be transmitted with moderate power. This study proposes a multi-level power adjustment (MLPA) mechanism for a wireless sensor network to prolong the lifetime of individual nodes and the overall network. In this study, we constructed an analytical model of the MLPA mechanism with $m$ distinct power levels ( $m$-LPA). For $m$-LPA, the closed-form expression of the optimal power setting was determined and the mean transmission power was minimized to one-third of the original fixed-transmission power. We found that the average power consumption of our proposed mechanism is 33.93\% higher than that of fixed-transmission power. Thus, each node can extend the lifetime by 2.5 times. We have shown the relations between $m$ and density in simulation results. Although the mechanism worked smoothly in this study, the sensors do not need to handle the distance and interference problem.
\end{abstract}

Keywords: Lifetime, Wireless sensor network, Power adjustment, Transmission power, Interference

\section{Introduction}

Power consumption has become one of the main topics in research on wireless sensor networks (WSNs). In the past few years, there has been much improvement in the technology and the application of WSNs [1-3]. Researchers have compiled complete sets of standards for real cases (e.g., IEEE 802.15.4 [4] and Zigbee) spanning a wide range of applications including home security, private sector organizations, health care, and the military. In general, each sensor has limited power and computing resources. Therefore, a major issue is the prolongation of network lifetimes for WSNs.

There are three basic subsystems on a sensor node: a sensing subsystem to collect data from the environment, a processing subsystem to provide computation ability to the sensor node, and a communication subsystem for the transmission of data [5]. These subsystems are all limited by internal sensor-node power. Each sensor node plays its part in performing sensing, computing, or transmitting, always within the context of limited energy. In order to extend the lifetime of WSNs, we focus our attention to transmission problems. Theoretically,

\footnotetext{
* Correspondence: jason0626@gmail.com

Department of Computer Science and Information Engineering, National Cheng Kung University, Tainan, Taiwan, Republic of China
}

transmission power can be separated into $n$ portions. In this study, we define the level is $n$ portions of transmission power. Each sensor must identify the number of poweradjustment level. And each sensor cans effective delivery packets to next node. In addition, each node should save transmission power efficiently. We propose a powercontrol mechanism that achieves efficient sensor-data transmission resting on two special features: (1) an adaptive transmission power rate for packet routing and (2) a prolongation of sensors' lifetime.

The major contributions of this article are as follows.

- We provide an multi-level power adjustment (MLPA) mechanism for implementation in a real WSN environment, and we prolong the lifetime of WSNs.

- We present a rigorous analysis of an MLPA mechanism and identify near-optimal power configurations for each power level.

- We present our findings that our proposed mechanism's (2LPA, 200-500 nodes) average power consumption registers a $33.93-55.30 \%$ improvement over the original fixed-transmission power method. Thus, the lifetime of each node can extend 2.5 times.

- As the simulation results show, we found that when we adjusted the power level to 2 or 3 , we could get 
acceptable performance. If the power level was more than 3 , the lifetime performance was better.

- The mechanism that we proposed can work smoothly even though to get the distance value between nodes and node is insufficient and even though there is some interference between nodes. In other words, the proposed mechanism can still work even if the between-node distance value is unsatisfactory, the sensor board lacks GPS chips, or the environment exhibits refraction and attenuation.

\section{Related work}

This section describes the literature's treatment of WSNs and various approaches to determining the transmission ranges for the extension of WSN lifetimes [6-10]. Conventional WSNs consist of thousands of sensor nodes and sensors that can communicate with each other or with base stations. The coverage area of a WSN depends on the number of sensors deployed [11-13].

Every sensor node has sensing, processing, transmitting, moving, locating, and electrical power elements; some elements are optional, such as the moving or locating element. Sensor nodes are placed in a particular area and integrated with each other to yield high-quality information about the environment [14]. Every sensor node not only can capture the sensed data and route it to other sensors or base stations, but also can determine methods of routing or scheduling according to the mission, the received data, and the status of the sensor node. A base station is a mobile or static device for connecting a WSN to the Internet, thereby enabling a user to receive sensed data.

In recent years, considerable research has addressed the coordination and management of sensing, as well as sensor-related data collection and processing [1]. Due to the limitations of both electrical power and sensor nodes' communication bandwidth, it is necessary to design a technology that reduces the waste of power and that employs limited bandwidth effectively. The limitations in traditional WSNs create many challenges of design and management such as electric power's relationship to each layer of a network's protocol stack. For example, it is necessary for the network layer to use a power-saving routing method that sends data from sensor nodes to the base station effectively and that increases the network's lifetime satisfactorily.

The routing methods of current WSNs include tabledriven, demand-driven, and hybrid methods. A detailed explanation of these three methods is given below.

(1) Table-driven method: According to the routing table in every sensor node, the sensed data can be transmitted by the default routing path in the routing table such as destination sequenced distance-vector (DSDV) [15], CGSR [16], and WRP [17].
(2) Demand-driven method: This method does not call for a default routing path. Instead, there is an ondemand path, which is found when source nodes must transmit data to a destination node. The routing path is established through flooding that involves node-path information from the source to the destination. AODV [18] and DSR [19] are examples of this kind of routing method.

(3) Hybrid method: This method is a combination of the table-driven and demand-driven methods. An example is ZRP [20].

Whereas at least three methods for solving problems of data transmission and of electrical power are based on routing, some approaches solve such problems by exploiting communication among sensor nodes [6-10,21,22]. These approaches, when successful, adjust communication power according to the between-node distance values [10], but they must solve problems concerning unknown transmission distance, uncertain range of sensor-node coverage, and nonlinear deployment in real WSNs.

Dhawan et al. [23] proposed a smoothly varying range model to adjust sensing range sensor networks for maximizing the network lifetime. Authors use the GargKönemann algorithm 1 with an approximation ratio to find a monitoring schedule for sensor covers. However, the continuous model needs complicate analysis. Wang and Medidi [24] proposed local sensing radii optimization schemes to minimize the energy consumption and extend the lifetime of networks. Authors assume that the transmission range is at least two times larger than the maximum sensing range, and then focus on optimizing sensing coverage for reliable surveillance and energyefficiency. However, this method requires additional mechanisms and overhead to ensure the connectivity of entire network if obstacles exist between sensors. In $[21,22]$, the authors divide the sensing field into a number of smaller areas. Based on those smaller area, sensors could control and minimizing energy consumption.

Because of these uncertainties and other unknowns, real WSNs cannot easily compute the precise communication power, even though some investigations have presented corresponding algorithms. This study proposes a power-adjustable routing policy, in which no sensor node transmits data by static communication power. Rather, each sensor node transmits data using dynamic communication power, determined by the conditions of the immediate environment.

\section{Multi-level power-adjustment mechanism}

In general, the required transmission power is the same for the communication between all sensor nodes and their neighboring nodes. Consider the example of the WSN shown in Figure 1. Regarding two pairs of sensor 


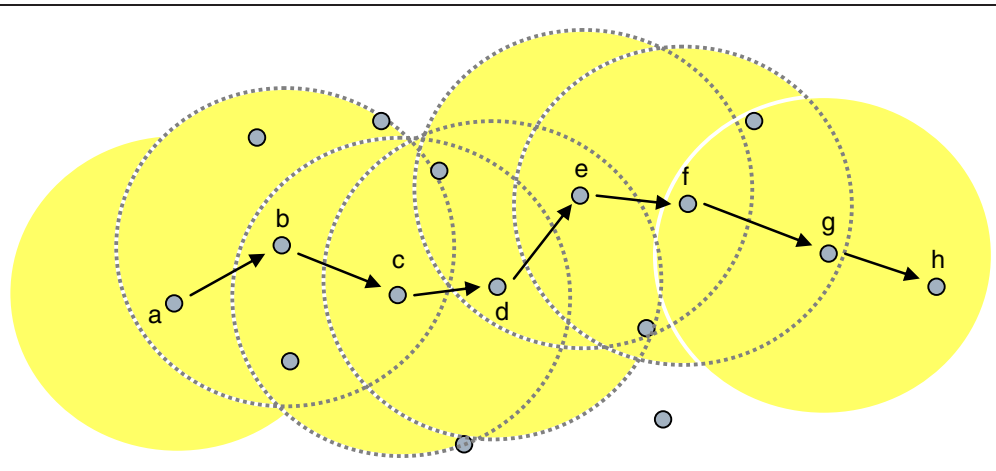

Figure 1 The situation of data-transmission power in traditional WSNs.

nodes, the distance between the first pair's two members differs from the distance between the second pair's two members, but the transmission power that consumes in sending data from one pair's member to the same pair's other member is the same for each pair. Consequently, the energy efficiency is unsatisfactory, because even if two sensor nodes are so close to each other that data can be transmitted using half the communication power (such as nodes c and d in Figure 1), the traditional method still transmits using the full communication power.

Adjustable transmission power is not a new concept. Lin et al. [25] presented adaptive transmission power control for WSNs, where each node constructs a model for each of its neighboring nodes that describe the relationship between transmission power and link quality. A feedbackbased transmission power control algorithm serves to maintain individual link quality over time dynamically.

This study presents a power-adjustment table (PAT) for every sensor node to determine the optimal communication power dynamically. Using the PAT, every sender node determines the communication power necessary for transmitting data and does so according to the environment of the nearby receiver node, the objective being to reduce power consumption. This method, accordingly, extends the
WSN lifetime. After the adaptive communication power is determined (as in Figure 2), each sender node uses a particular communication power to transmit data consistent with the environment of the nearby receiver node.

In the proposed MLPA mechanism, the transmission corresponding to each sensor node involves adjustable power. The key feature of MLPA is the use of just enough power to communicate with neighboring nodes. Less power is used for closer nodes. The MLPA mechanism operates according to the following three phases.

- Phase I: Neighbor discovery and construction of network topology

Initially, each node broadcasts a beacon to identify all possible neighboring nodes. Full transmission power functions to maximize the network connectivity level.

- Phase II: Negotiation and adjustment of transmission power negotiation

Energy is conserved by reducing the average transmission power. Full power is not necessary for transmission to a nearby neighbor. In this phase, a sensor node negotiates with its neighboring nodes and determines which power level should

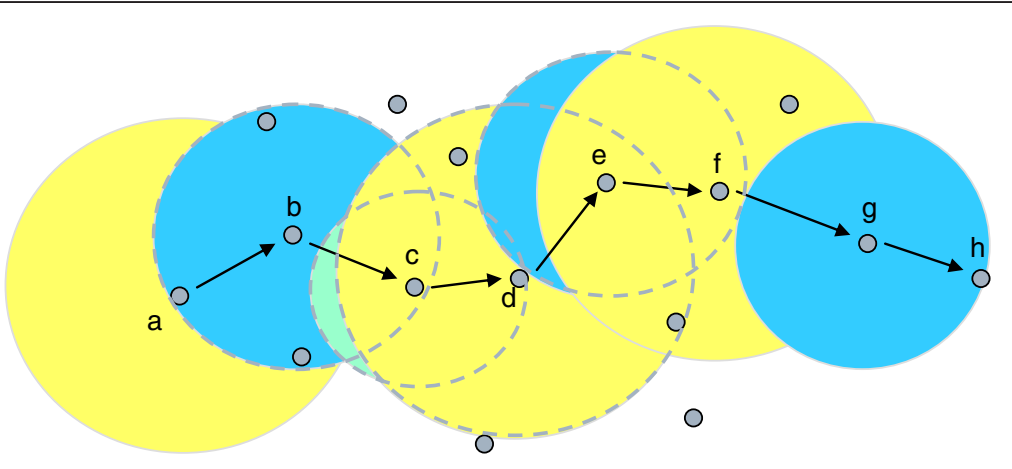

Figure 2 The data transmission after determination of the adaptive communication power. 
correspond to each neighbor. Given the negotiation overhead, the piggyback mechanism can function to reduce the overhead involved in power negotiation.

- Phase III: Runtime maintenance

The communication environment may change dynamically after node deployment. In addition, the movement of nodes may change network topology. Sensor nodes must repeat phases I and II to update the network topology and preserve the optimal power assignment for each neighbor.

We use MLPA mechanisms to improve energy conservation. Our mechanism and analysis can be divided into two parts: (1) multi-level construction of a PAT; (2) optimal multi-level power-configuration analysis.

\section{Multi-level construction of a PAT}

For this study, we initially assumed that each sensor node would have the ability to adjust its own power. Theoretically, transmission power can be separated into $n$ portions, and each sensor must identify the number of power-adjustment level. And each sensor scans effective delivery packets to next node. In addition, each node should save transmission power efficiently. There are some solutions which will precisely compute transmission power by distance. The sensors always use maximum transmission power to deliver packets because sensors have no internal GPS chip for computing the distance between each node and because real environments involve significant interference and attenuation. In this study, we propose a mechanism to solve this problem. We assume that transmission power is separated into three portions: $\mathrm{L}, \mathrm{M}$, and $\mathrm{S}$. We use this assumed scenario to explain
MLPA construction. Figure 3 shows the coverage of these three portions of transmission power.

In the WSN's initial stage, each sensor node will transmit on the basis of these three power discover messages in order to collect neighboring nodes' information. When we use transmission power $L$ to transmit a discover message, we collect neighboring nodes' information in power $L$ coverage area. And when we use transmission power $M$ to transmit a discover message, we collect neighboring nodes' information in power $M$ coverage area. This is the pattern that repeats itself. Each sensor node transmits these three powers in the WSN's initial stage and, if successful, senses other neighbors' sensors in its coverage area. These data are stored in a PAT, as given below.

The PAT is stored in the MAC layer. When the routing protocol goes into operation to determine the next transfer node of a packet, our proposed mechanism picks an appropriate power level for this packet in the MAC layer. This study transmits packets by the transmission power level in the physical layer. Through this PAT, we can determine a node's neighboring node, transmission power consumption, the operating (alive) status of the node, and the response status of the node. A sensor node can optimize transmission power using this PAT to complete the transmission.

As shown in Figure 4, node $i$ transmits data through node $k$ to node $j$. Table 1 presents our assumed scenario involving a PAT for node $k$. When node $k$ receives data from node $i$, node $k$ already knows that the next node is node $j$. Hence, node $k$ uses level- $M$ transmission power to transmit data to node $j$, after querying the PAT for node $k$.

When a WSN is deployed in a real environment, measuring the distance between nodes is difficult. Even if the distance between sensor nodes is known, avoiding

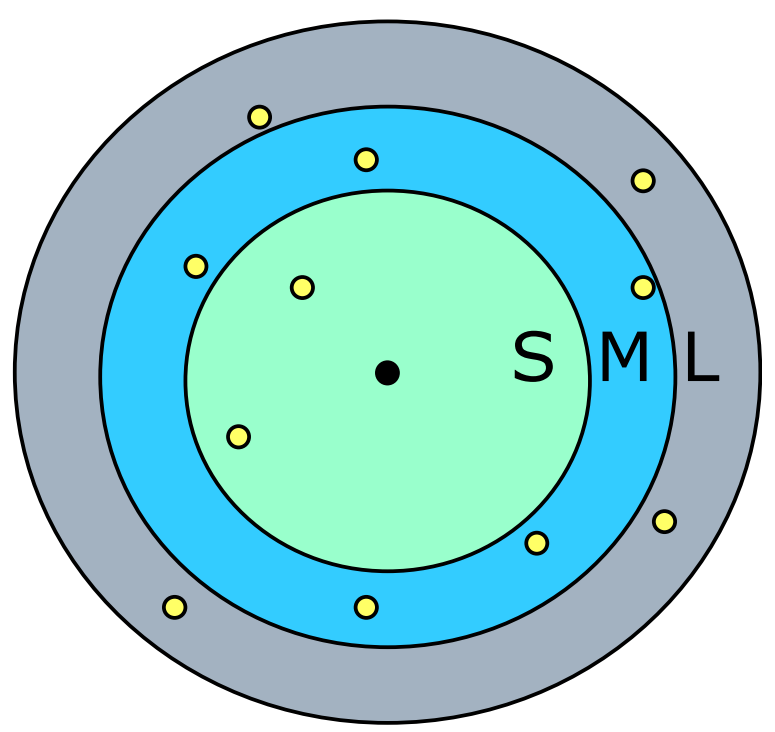

Figure 3 Different transmission powers with different signal coverage. 


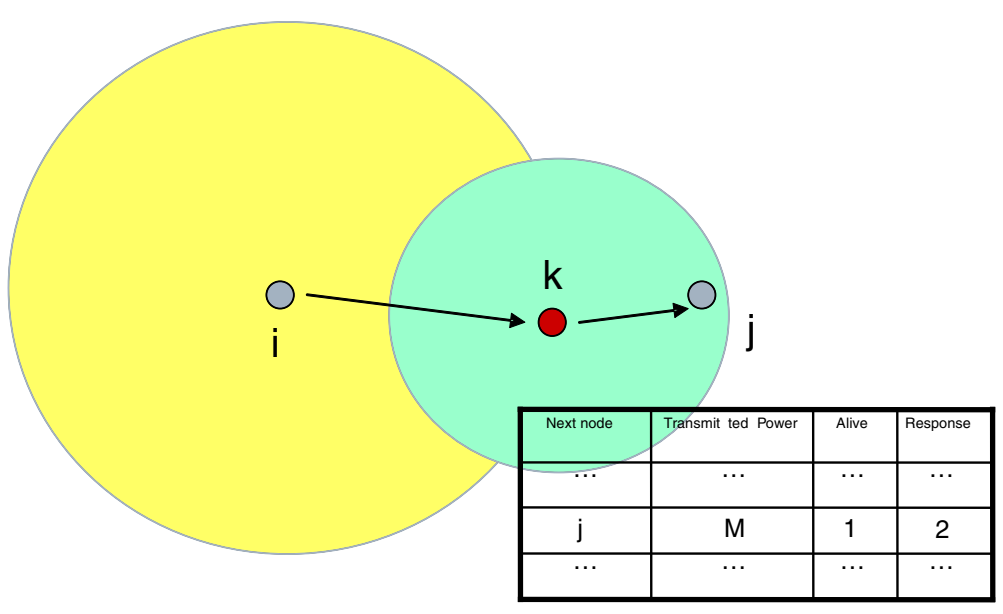

Figure 4 Use of appropriate transmission power through a PAT.

interference and the effects of nearby obstacles is difficult. Accordingly, using mathematical analysis to calculate transmission power accurately is almost impossible. However, the mechanism proposed herein can take into account environmental conditions between sensor nodes. Consequently, every sensor node can transmit data with optimal power.

\section{Optimal multi-level power-configuration analysis}

Our analysis reveals that a WSN can reduce the energy consumed by transmission through an adaptive power-routing strategy. The ratio of retrenched energy consumption is based on the levels of transmission power. These affect the energy efficiency in a WSN. This section will analyze the specific value of transmission power in order to determine the optimal levels of transmission power.

Each sensor node differently adjusts the transmission power. Multiple transmission power adjustments must be made in the situation represented by Figure 5 . Theoretically, transmission power can be cut into $m$-LPA. This study discusses the example of a 3-LPA power-adjustment method, and makes the following assumptions.

(1) $N$ sensor nodes are randomly deployed over an area, $A$. The node density $(\rho)$ is defined as $\rho=N / A$.

Table 1 Example of a PAT in a sensor node

\begin{tabular}{cccc}
\hline Neighboring node & Transmitted power & Alive & Response \\
\hline$a$ & $L$ & 1 & 1 \\
$j$ & $M$ & 1 & 4 \\
$e$ & $M$ & 0 (dead) & (null) \\
$g$ & $M$ & 2 (asleep) & 3 \\
$f$ & $M$ & 1 & 4 \\
$i$ & $S$ & 1 & 1 \\
\hline
\end{tabular}

(2) For an arbitrary node, the transmission probabilities to all neighboring nodes are equal.

(3) Assume that a signal is attenuated by distance $d$ raised to the power $\beta$, where $\beta$ is the path-loss exponent (PLE). The power of the intended signal from the transmitter at the receiver can be written as [26]

$$
\begin{aligned}
& P_{r}=\frac{\alpha P_{t}}{d^{\beta}} \\
& \alpha=\frac{G_{t} G_{r} \lambda^{2}}{(4 \pi)^{2}}
\end{aligned}
$$

where $P_{t}$ stands for the transmission power at the transmitting antenna, $P_{r}$ denotes the signal power measured at the receiving antenna, $G_{t}$ and $G_{r}$ denote the transmitter and receiver antenna gains, and $\beta$ denotes the wavelength of the carrier. The PLE depends on the wireless environment and typically varies from two to four. Initially in the free space loss (FSL) model, PLE $\beta=2$ is considered.

The largest transmission power, $P$, is determined. Then, the maximum transmission radius $R$ can be determined. Without any power adjustment, the sensor nodes transmit data using $P$, in line with the above statements. Various transmission radii can be derived, $r_{1} R, r_{2} R, \ldots$, $r_{m-1} R, r_{m} R$, where $0<r_{1}<r_{2}<\cdots<r_{m-1}<r_{m}=1$, for a sensor node that makes an $m$-level transmission power adjustment, and the relation between transmission power and transmission distance can be obtained through an FSL model.

$$
P_{i}=r_{i}^{2} P, i=1,2, \ldots, m .
$$

When a sensor node transmits data using transmission power $P_{i}$, the sensor nodes at distances between $r_{\mathrm{i}-1} R$ 


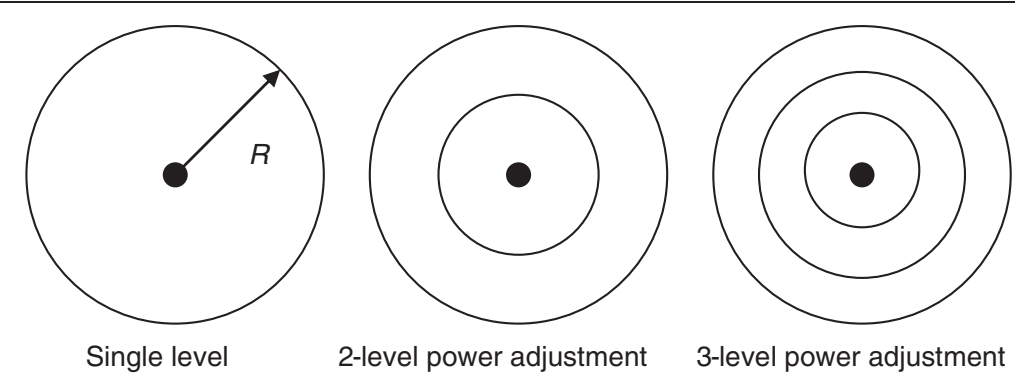

Figure 5 MLPA.

and $r_{\mathrm{i}} R$ can receive the data. Therefore, the number of sensor nodes in the range is

$$
n_{i}=\rho \pi\left(r_{i}^{2}-r_{i-1}^{2}\right) R^{2}, \text { for } i=2,3, \ldots, m .
$$

All sensor nodes are assumed to have equal transmission probabilities and access. Therefore, the equation that governs the $m$-level adjustment at the average power consumption is as follows.

$$
P_{a v g(m)}=\frac{\sum_{i=1}^{m} P_{i} n_{i}}{\sum_{i=1}^{m} n_{i}}
$$

The 3-LPA power adjustment proposed in this study yields three transmission ranges.

$$
r_{1} R, r_{2} R \text {, and } r_{3} R \text {, where } 0<r_{1}<r_{2}<r_{3}=1 \text {. }
$$

Transmissions in the three ranges are received according to the following transmission power ratios.

$$
\begin{aligned}
& P_{1}=r_{1}^{2} P \\
& P_{2}=r_{2}^{2} P \\
& P_{3}=P
\end{aligned}
$$

The above ratios and distances yield the number of nodes in each transmission range.

$$
\begin{aligned}
& n_{1}=\rho \pi\left(r_{1} R\right)^{2} \\
& n_{2}=\rho \pi\left(r_{2} R\right)^{2}-\rho \pi\left(r_{1} R\right)^{2}=\rho \pi R^{2}\left(r_{2}{ }^{2}-r_{1}^{2}\right) \\
& n_{3}=\rho \pi R^{2}-\rho \pi\left(r_{2} R\right)^{2}=\rho \pi R^{2}\left(1-r_{2}^{2}\right)
\end{aligned}
$$

Then, the average transmission power consumption is calculated as follows.

$$
\begin{aligned}
P_{\text {avg }(3)} & =\frac{P_{1} \cdot n_{1}+P_{2} \cdot n_{2}+P_{3} \cdot n_{3}}{n_{1}+n_{2}+n_{3}} \\
& =\left\{r_{1}^{4}+\left(r_{2}^{2}-r_{1}^{2}\right) r_{2}^{2}+\left(1-r_{2}^{2}\right)\right\} P
\end{aligned}
$$

Therefore, to minimize the average transmission power, not only must one minimize $f\left(r_{1}, r_{2}\right)$, but also both $r_{1}$ and $r_{2}$ must satisfy $0<r_{1}<r_{2}<r_{3}=1$.
Figure 6, produced in MATLAB, is a threedimensional representation of the transmission power ratio for $r_{1}, r_{2}$, and the average transmission power consumed.

On the basis of the above figure, one can find an ideal power ratio by using differential calculus to minimize 3-LPA:

$$
\begin{aligned}
& f_{r_{1}}\left(r_{1}, r_{2}\right)=\frac{\partial f\left(r_{1}, r_{2}\right)}{\partial r_{1}}=4 r_{1}^{3}-2 r_{1} r_{2}^{2} \\
& f_{r_{2}}\left(r_{1}, r_{2}\right)=\frac{\partial f\left(r_{1}, r_{2}\right)}{\partial r_{2}}=-2 r_{2}+4 r_{2}^{3}-2 r_{2} r_{1}^{2}
\end{aligned}
$$

The power ratios at $r_{1}$ and $r_{2}$ are obtained as follows.

$$
\begin{aligned}
& \left\{\begin{array}{r}
-2 r_{2}^{2} r_{1}+4 r_{1}^{3}=0 \\
-2 r_{2}+4 r_{2}^{3}-2 r_{2} r_{1}^{2}=0
\end{array}\right. \\
& r_{1}=\frac{\sqrt{3}}{3} \quad r_{2}=\frac{\sqrt{6}}{3}
\end{aligned}
$$

Therefore, the 3-LPA power ratio for $L: M: S$ is 1 : $\left(\sqrt{6} /{ }_{3}\right):\left(\sqrt{3} /{ }_{3}\right)$. These calculations enable one to obtain the average transmission power consumption $P_{\text {avg }}^{\min }(3)=(2 / 3) P$ at $r_{1}=\sqrt{3} / 3$ and $r_{2}=\sqrt{6} / 3$. The minimum average transmission power is $(2 / 3) P$ for 3 -LPA in an FSL environment. The optimal configuration is as follows.

$$
\left\{\begin{array} { l } 
{ P _ { 1 } = ( 1 / 3 ) P } \\
{ P _ { 2 } = ( 2 / 3 ) P } \\
{ P _ { 3 } = P , }
\end{array} \left\{\begin{array} { l } 
{ R _ { 1 } = ( \sqrt { 3 } / 3 ) R } \\
{ R _ { 2 } = ( \sqrt { 6 } / 3 ) R } \\
{ R _ { 3 } = R , \text { and } }
\end{array} \left\{\begin{array}{l}
n_{1}=\rho \pi R^{2} / 3 \\
n_{2}=\rho \pi R^{2} / 3 \\
n_{3}=\rho \pi R^{2} / 3
\end{array}\right.\right.\right.
$$

The above analysis reveals that appropriately setting the specific value of transmitter power can reduce the transmitter power to a level that is one-third less than that of the fixed-transmission power method.

\section{Optimal power configuration}

For the case of PLE $\beta \neq 2$, the power configuration of each power level corresponds to a closed-form expression. 


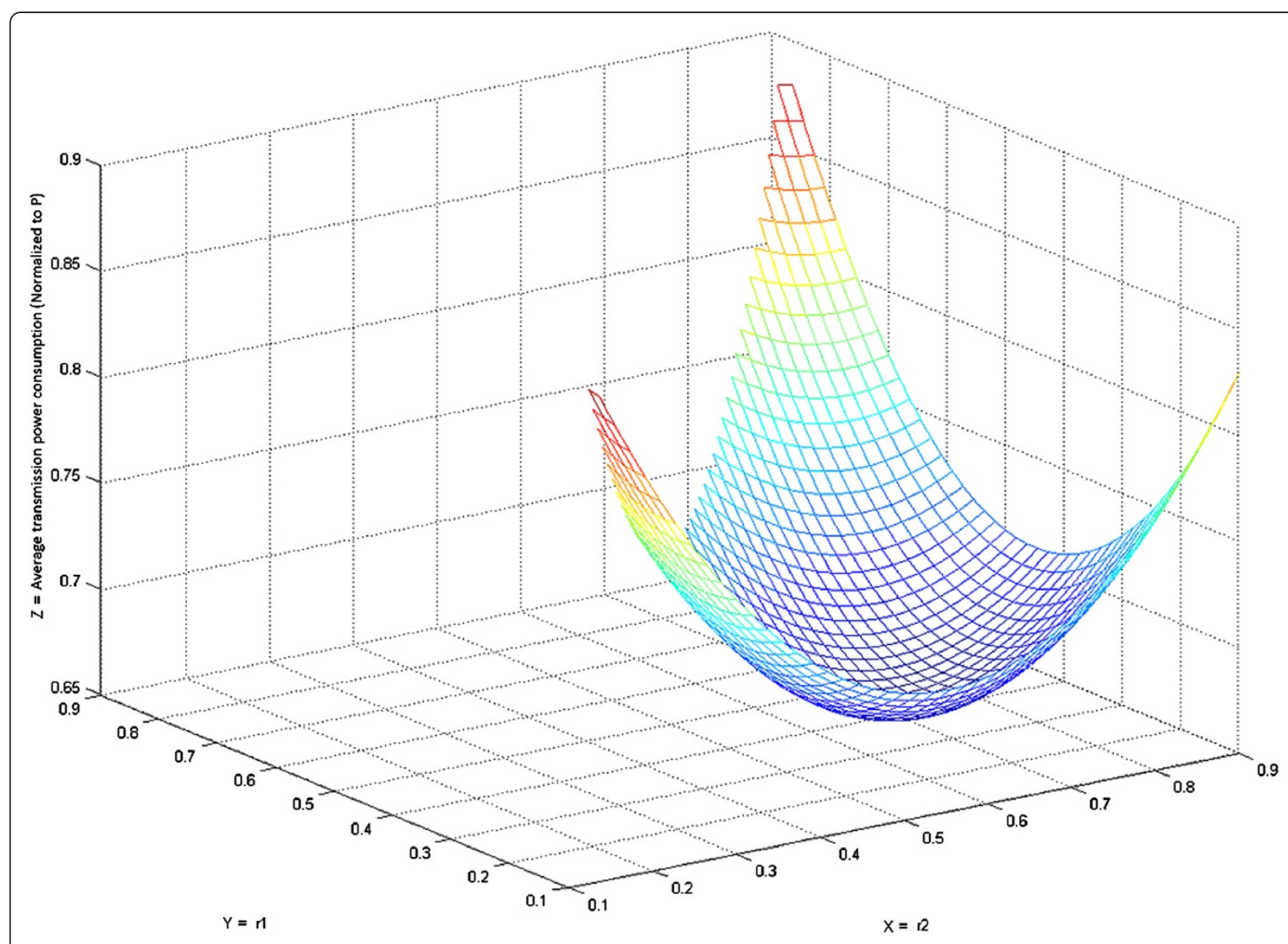

Figure $6 \mathrm{~A}$ diagram of the transmission power ratio and the average transmission power consumption relative to 3-LPA.

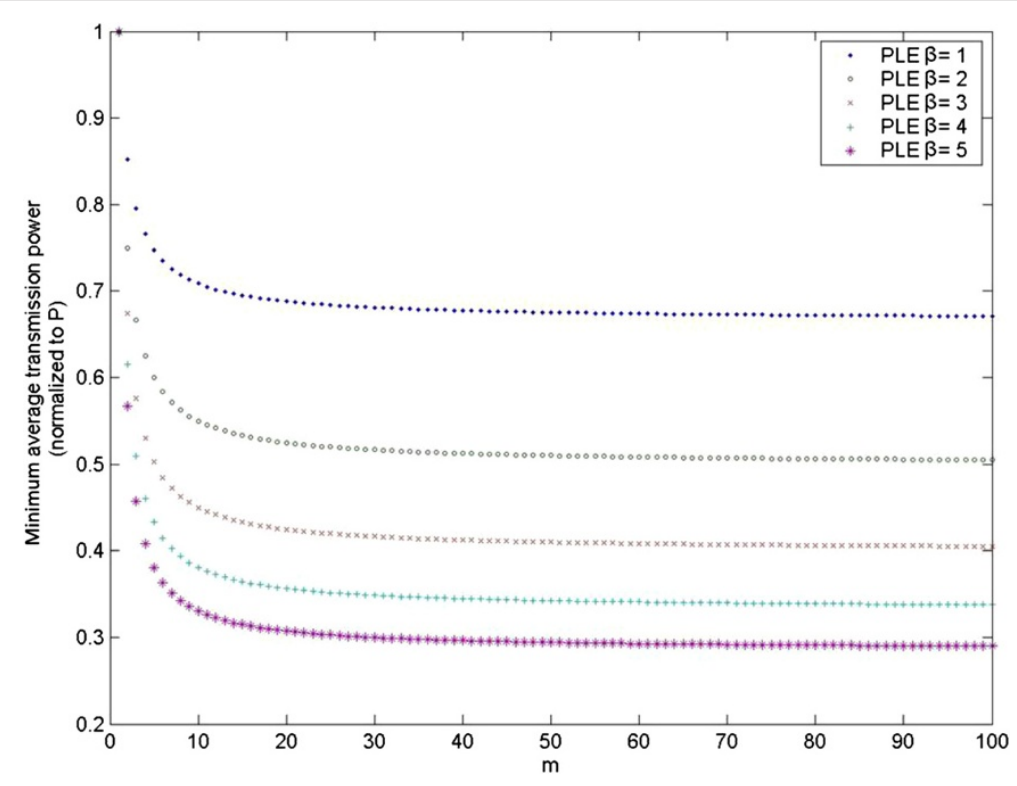

Figure 7 Minimum average transmission power for $m$-LPA. 
Table 2 Optimal configuration for $m$-LPA

\begin{tabular}{lcccccl}
\hline & $\boldsymbol{r}_{\mathbf{1}}$ & $\boldsymbol{r}_{\mathbf{2}}$ & $\boldsymbol{r}_{\mathbf{3}}$ & $\boldsymbol{r}_{\mathbf{4}}$ & $\boldsymbol{r}_{\mathbf{5}}$ & $\boldsymbol{P}_{\text {avg }}^{\min }(\boldsymbol{m})$ \\
\hline$m=2$ & $\sqrt{\frac{1}{2}}$ & 1 & - & - & - & $\frac{3}{4} P$ \\
$m=3$ & $\sqrt{\frac{1}{3}}$ & $\sqrt{\frac{2}{3}}$ & 1 & - & - & $\frac{2}{3} P$ \\
$m=4$ & $\sqrt{\frac{1}{4}}$ & $\sqrt{\frac{1}{2}}$ & $\sqrt{\frac{3}{4}}$ & 1 & - & $\frac{5}{8} P$ \\
$m=5$ & $\sqrt{\frac{1}{5}}$ & $\sqrt{\frac{2}{5}}$ & $\sqrt{\frac{3}{5}}$ & $\sqrt{\frac{4}{5}}$ & 1 & $\frac{3}{5} P$ \\
\hline
\end{tabular}

The minimum average transmission power of $m$-LPA is given by the following recursive formula.

$$
\begin{aligned}
& P_{\text {avg }}^{\min }(m, \beta)
\end{aligned}
$$

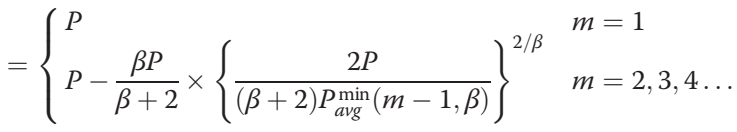

The recursive formula can be verified by mathematical induction. The power configuration of each level depends on the value of $r_{i}$, which can be described as

$$
r_{i}= \begin{cases}\prod_{j=i}^{m-1} q_{j} & 1 \leq i<m \\ 1 & i=m\end{cases}
$$

where $q_{j}=\frac{r_{j}}{r_{j+1}}=\left\{\frac{2 P}{(\beta+2) P_{a v g}^{\min }(j, \beta)}\right\}^{1 / \beta}$. When the value of $r_{i}$ is computed, the transmission power of sensor can be computed as follows.

$$
P_{i}=r_{i}^{\beta} P
$$

$$
R_{i}=r_{i} R^{\prime}
$$

where $R^{\prime}=R^{2 / \beta}$ is the maximum transmission range at power $P$ in the wireless environment of PLE $\beta$.

Figure 7 plots the minimum average transmission power versus the degree of MLPA. The number of power levels varies from 1 to 100 . At $m=100$, the minimum average transmission powers are $0.671,0.505,0.405,0.338$, and 0.29 for PLE $\beta=1,2,3,4$, and 5 , respectively.

\section{Near-optimal power configuration}

As the degree of $m$-LPA increases, manually optimizing the configuration grows difficult. The built-in function "Minimize" in Mathematica [27], a powerful scientific and mathematical software package, thus serves to minimize $P_{\text {avg }}(m)$, subject to the constraint $0<r_{1}<r_{2}<\cdots$ $<r_{m}=1$. Table 2 presents the optimal configuration and the minimum average transmission power for $m$-LPA, as $m$ varies from two to five.

Given Table 2, we suggest a closed-form expression for the optimal configuration for $m$-LPA, where $m$ is a positive integer. In the optimal power configuration of $m$-LPA (which minimizes the average transmission power), the $r_{i}$ configurations are conjectured to be

$$
r_{i}=\sqrt{i / m}
$$

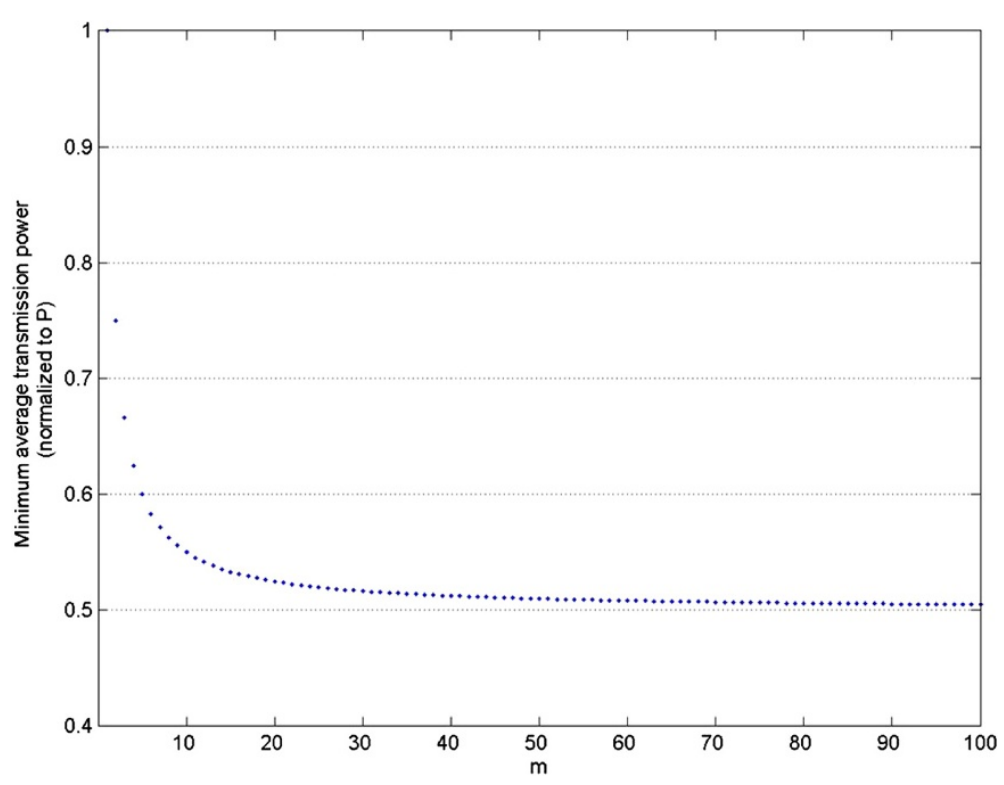

Figure 8 Minimum transmission power for $m$-LPA $(\beta=2)$. 


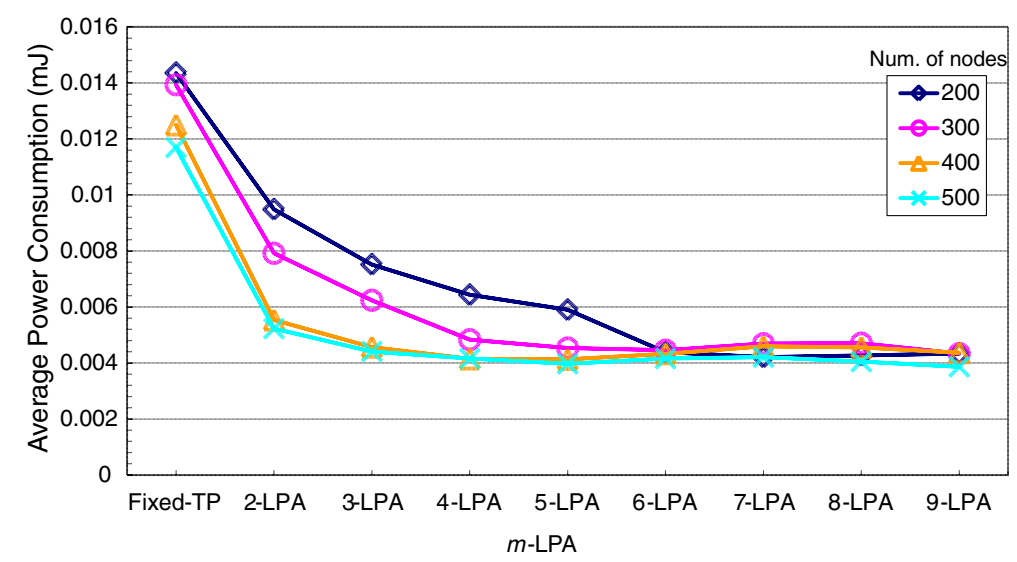

Figure 9 Average power consumption for $m$-LPA (200-500 nodes).

Consequently, each transmission power is described as

$$
P_{i}=\frac{i}{m} P
$$

When $m$-LPA is used, the average transmission power can be minimized to

$$
P_{\text {avg }}^{\min }(m)=\frac{m+1}{2 m} P .
$$

Figure 8 plots the minimum average transmission power versus the degree of MLPA $(m)$. As shown in Figure 8 , the minimum average transmission power is a monotonically decreasing function of $m$. Equation (24) reveals that a higher degree of MLPA corresponds to a greater transmission power saving. The limit of $P_{a v g}^{\min }(m)$ $P$ is reached as $m$ approaches infinity.

$$
\lim _{m \rightarrow \infty} P_{\text {avg }}^{\min }(m)=\frac{P}{2} .
$$

Ideally, half of the transmission power can be saved as the number of power levels approaches infinity if the optimal power is used for transmission to each neighboring node. However, providing an MLPA mechanism with an infinite number of power levels is both unfeasible and unnecessary. The power negotiation overhead must also be considered. For $m=10$, the maximum transmission-power savings are $45 \%$, which is $90 \%$ of the maximum possible power savings in the FSL model. In general, the use of optimized $m$-LPA yields a transmission power savings of $\frac{100(m-1)}{2 m} \%$, which is $\frac{100(m-1)}{m} \%$ of the maximum possible power savings in the FSL model.

\section{Performance analysis and results}

We wrote a simulation program to confirm the MLPA mechanism; and we compared static transmission power and transmission under the MLPA mechanism. Adjusting transmission power according to the proposed mechanism

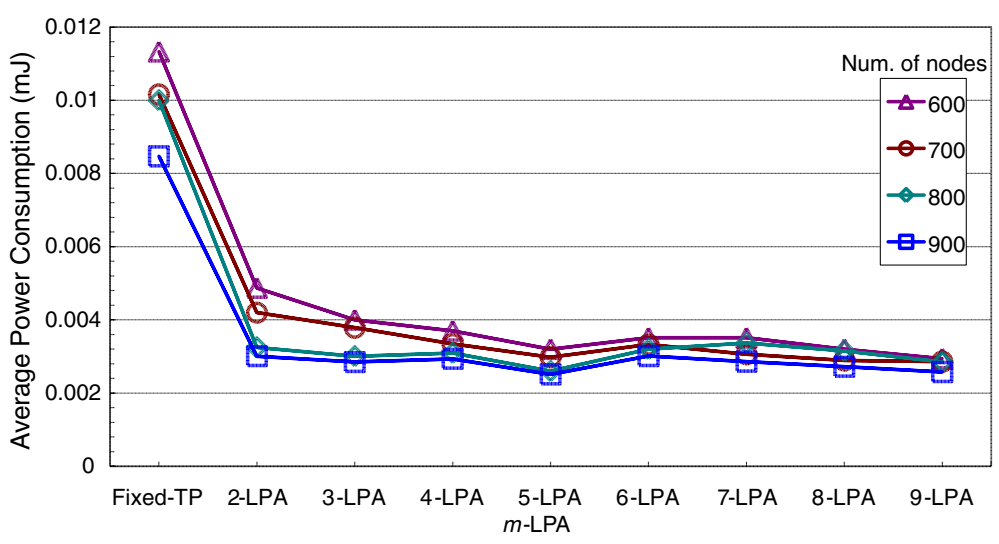

Figure 10 Average power consumption for $m$-LPA (600-900 nodes). 


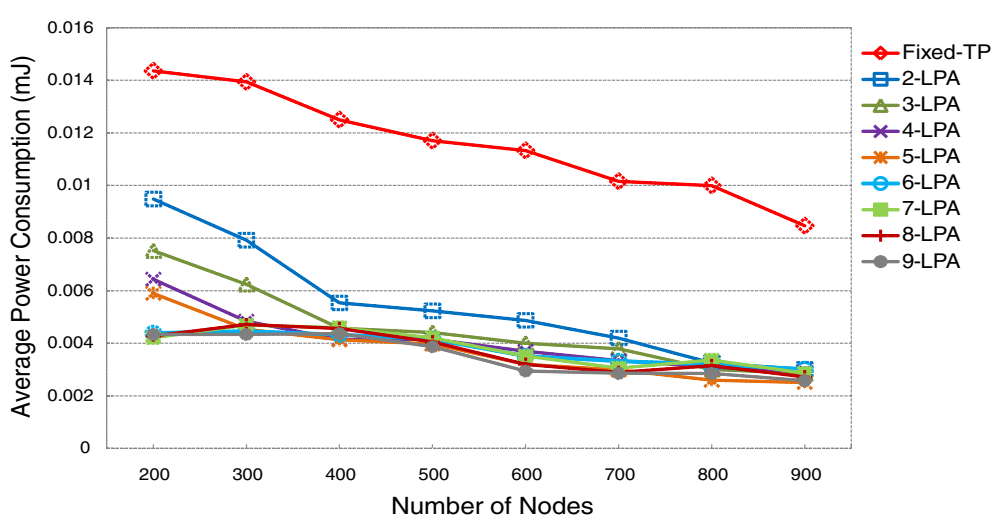

Figure 11 Average power consumption of sensor node with $m$-LPA and Fixed-TP.

enables the packets to arrive at the sink node, but reduces the power consumption by almost half.

When the MLPA mechanism is applied, the transmission power undergoes adaptive changes to deliver packets to the next node. The routing protocol is assumed to be tabledriven. When routing data, the sensor node looks up the routing table. In the simulation, DSDV [15] is used as the routing protocol. We compared the proposed mechanism to the one that uses fixed transmission power (Fixed-TP).

To determine the efficiency of the MLPA mechanism, we performed a simulation using Visual $\mathrm{C}++$. The sensor area was set to $600 \times 600 \mathrm{~m}^{2}$. The number of deployed sensor nodes ranged between 200 and 900 . The transmission range of the sensor was $50 \mathrm{~m}$. When the simulation ran, if the number of sensor nodes was under 200, the sensor network could not maintain its network connectivity and WSN failed to cover some regions. Therefore, at least 200 sensor nodes are needed to achieve adequate network functioning.

The DSDV was used as the routing protocol. DSDV is an enhanced version of the distributed Bellman-Ford algorithm. In DSDV, each node maintains the routing table that includes (1) the shortest distance between nodes and sink and (2) the first node on the shortest path to every other node in the network. DSDV includes table updates with increasing numbers of sequence tags to prevent loops.

In this article, we refer to T-mote sensors set to specific power parameters. Simulation program assigns the packet-generation probability in accordance with Poisson probability. In the simulation, we compared the MLPA mechanism ( $m=2$ to 9 ) with the traditional Fixed-TP mechanism, which uses only a simple kind of power to deliver information $(m=1)$. Figure 9 shows that we saved an average of more than $47 \%$ of the average power consumption regarding the low-density group (number of nodes between 200 and 500), and Figure 10 shows that we saved an average of nearly $62 \%$ of the average power consumption regarding the high-density group (number of nodes between 600 and 900).

Figure 9 shows the average power consumption of WSNs when (1) the effect of $m$ from 2 to 6 is greater than that of $m$ on over 6 and (2) when $2<m<6$. The comparison between the scheme with Fixed-TP and

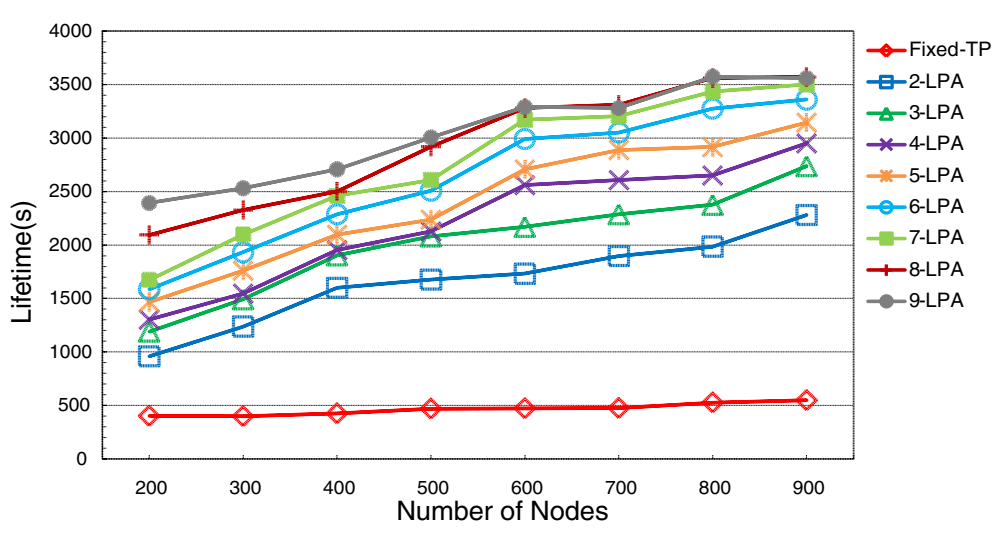

Figure 12 Lifetime(s) of WSN with $m$-LPA and Fixed-TP. 


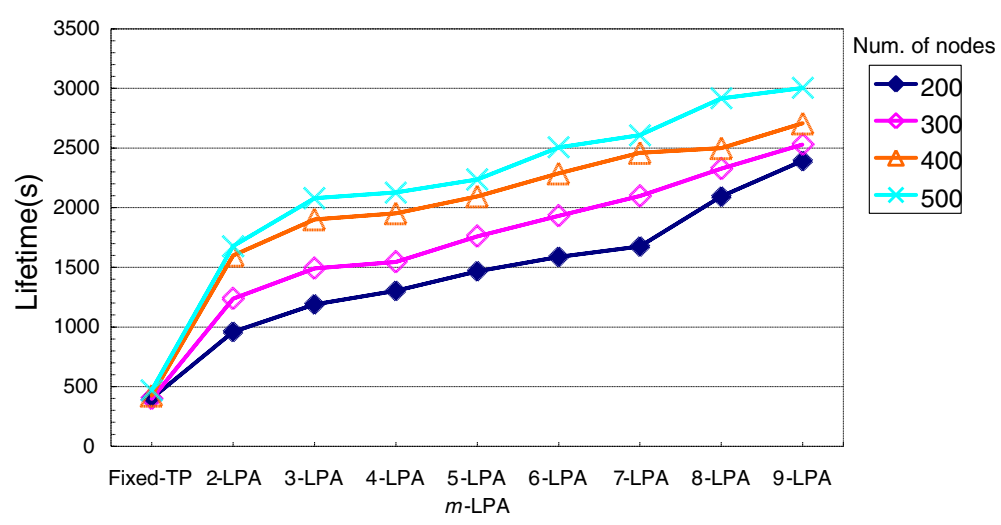

Figure 13 Lifetime(s) of WSN with $m$-LPA (200-500 nodes).

MLPA scheme with 2 LPA shows that our scheme saves 33.93-55.30\% energy under 200-500 nodes in Figure 9. The average power consumption saves $47.04 \%$ under sparse environment in summary. Figure 10 shows the average power consumption for efforts to strengthen the effect of $m$ from 2 to 3 . The average power consumption saves $61.92 \%$ under density environment (600-900 nodes). Furthermore, transmission energy consumption can be saved up to $69.61 \%$ using MLPA scheme with 9 LPA.

Figure 11 shows the $m$-LPA compared with the Fixed-TP regarding average power consumption. A sensor node's average power consumption for Fixed-TP was $0.01436 \mathrm{~mJ}$ in a 200-node environment, but in the same environment, the 2-LPA set up needed just $0.009487 \mathrm{~mJ}$; for the 9-LPA set up, the amount needed was only $0.004338 \mathrm{~mJ}$. Our proposed method is better than Fixed-TP when the objective is to significantly lower the average power consumption.

Figure 12 shows the lifetimes of WSN with $m$-LPA, and the figure compares our proposed mechanism with Fixed-TP. Fixed-TP's use of full power to transmit every packet will result in a shortened lifetime for WSNs. The MLPA method allows for the selection of different types of transmission power to deliver packets, and in this regard, Figure 12 presents some interesting findings. MLPA's lifetime turned out to be much longer than Fixed-TP's lifetime.

Figures 13 and 14 show that the Fixed-TP lifetime are short. We compared the lifetimes of the MLPA mechanism with those of the 2-LPA and Fixed-TP approaches, and found that 2-LPA had an average extension of 2.5 times the lifetimes of WSNs. In Figure 13, we can see that when the density of deployment is low, lifetimes under the MLPA mechanism $(2<m \leqq 9)$ can be extended. And Figure 14 shows that when $m=2$ and 3 , lifetimes get a boost. The growth in lifetimes is less pronounced than the growth when $m>3$.

From Figure 15, we can also understand the effect that density has on lifetimes. The Fixed-TP performance is far below the $m$-LPA $(m>1)$ performance. And when the density is less than 0.17 and $m>4$, lifetime performance is quite remarkable, but when the density is greater

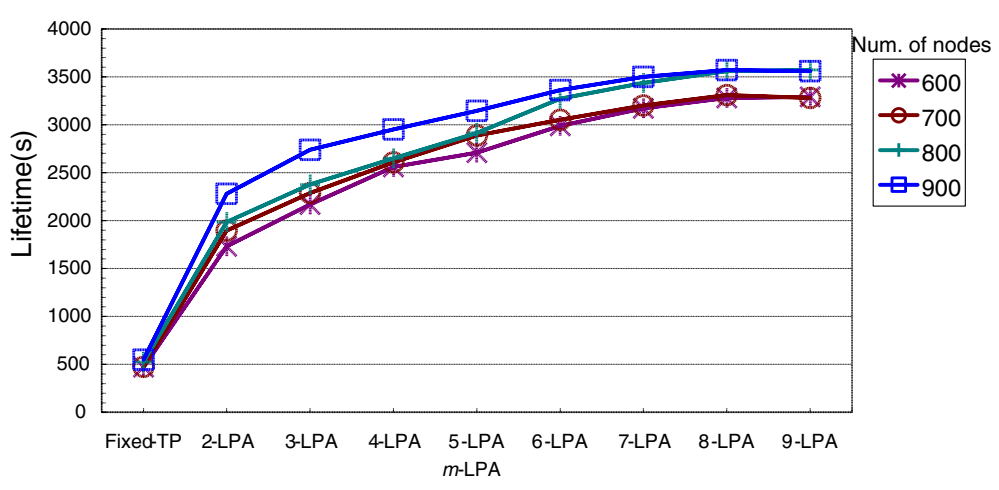

Figure 14 Life time of WSN with $m$-LPA (600-900 nodes). 


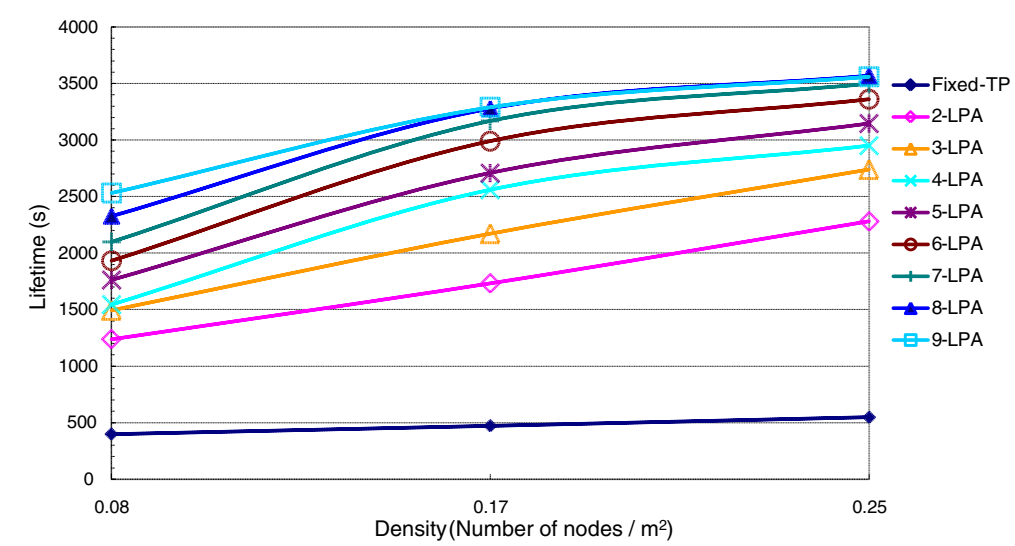

Figure 15 Relationship between lifetimes of WSN and density.

than 0.17 and $m>4$, the lifetime curve is flat. For different density environments, generally increasing the $m$ value will enhance and improve the lifetime of whole WSNs. Figure 15 shows that performance tends to get overlap when $m=8$ and 9 in situations where the density is greater than 0.17 .

Therefore, any effort to set the $m$ value in relation to the environment must account for the density of deployment. In general, $m=2$ or 3 can yield a pretty good performance.

\section{Conclusion}

We proposed an MLPA mechanism for implementation in real WSN environments to prolong the lifetime of WSNs. And we present a rigorous analysis of an MLPA mechanism and identify near-optimal power configurations for each power level. On the basis of PAT construction, appropriate transmission power can be adjusted during data transmission. We present our findings that the average power consumption of our proposed mechanism (2LPA, 200-500 nodes) registers a 33.93-55.30\% improvement over the original Fixed-TP method. Thus, the lifetime of each node can extend 2.5 times. And we have presented the relations between $m$ and density in simulation results. Setting the appropriate $m$ value will improve lifetime performance.

As the simulation results show, we found that when we adjusted the power level to 2 or 3 , we could get acceptable performance. If the power level was more than 3 , the lifetime performance was better.

The mechanism that we proposed can work smoothly even though to get the distance value between nodes and node is insufficient and even though there is some interference between nodes. In other words, the proposed mechanism can still work even if the betweennode distance value is unsatisfactory, the sensor board lacks GPS chips, or the environment exhibits refraction and attenuation.
We conclude that the proposed mechanism can work smoothly. If those sensors cannot get the distance between nodes and there is some interference between nodes, our proposed mechanism can work smoothly.

\section{Competing interests}

The authors declare that they have no competing financial interests.

Received: 16 December 2011 Accepted: 4 October 2012

Published: 16 November 2012

\section{References}

1. W Ye, J Heidemann, D Estrin, Medium access control with coordinated adaptive sleeping for wireless sensor networks. IEEE/ACM Trans. Netw. 12(3), 453-506 (2004)

2. T van Dam, $\mathrm{K}$ Langendoen, An adaptive energy-efficient MAC protocol for wireless sensor networks, in 1st ACM Conference on Embedded Networked Sensor Systems(ACM SenSys), ed. by (Los Angeles, CA, 2003), pp. 171-181

3. S Tilak, NB Abu-Ghazaleh, W Heinzelman, Infrastructure tradeoffs for sensor networks, in Proceedings of the 1st ACM International Workshop on Sensor Networks and Applications (WSNA), ed. by (Atlanta, 2002), pp. 49-58

4. IEEE Std 802.15.4, Part 15.4, Wireless medium access control (MAC) and physical layer (PHY) specification for low rate wireless personal area networks (LR-WPANs). (2003)

5. RA Santos, L Villasenor, J Gallardo, A Edwards, Performance analysis of routing strategies for wireless sensor networks. IFIP 248, 191-202 (2007)

6. E Althaus, G Calinescu, II Mandoiu, S Prasad, N Tchervenski, A Zelikovsky, Power efficient range assignment for symmetric connectivity in static ad hoc wireless networks. Wirel. Netw 12, 287-299 (2006)

7. M Huang, PE Caines, RP Malhamé, Uplink power adjustment in wireless communication systems: a stochastic control analysis. IEEE Trans. Automatic Control 49(10), 1693-1708 (2004)

8. S Panichpapiboon, G Ferrari, OK Tonguz, Optimal transmit power in wireless sensor networks. IEEE Trans. Mob. Comput. 5, 1432-1447 (2006)

9. R Ramanathan, R Rosales-Hain, Topology control of multihop wireless networks using transmit power adjustment, in Proc. IEEE Infocom'2000, ed. by (Tel Aviv, Israel, March 2000), pp. 404-413

10. Q Gao, KJ Blow, DJ Holding, IW Marshall, XH Peng, Radio range adjustment for energy efficient wireless sensor networks. Ad Hoc Netw. 4(1), 75-82 (2006)

11. IF Akyildiz, W Su, Y Sankarasubmmaiam, E Cayirci, A survey on sensor networks. IEEE Commun. Mag. 40(8), 102-114 (2002)

12. JN Al-Karaki, AE Kamal, Routing techniques in wireless sensor networks: a survey. IEEE Wirel. Commun. 11, 6-28 (2004)

13. C Intanagonwiwat, D Estrin, R Govindan, J Heideman, Impact of network density on data aggregation in wireless sensor networks, in International Conference on Distributed Computing Systems, ed. by (Vienna, 2002), pp. $457-458$ 
14. Y Gao, K Wu, F Li, Analysis on the redundancy of wireless sensor networks, in Proc. 2nd ACM International Workshop on Wireless Sensor Networks and Applications (WSNA), ed. by (San Diego, 2003), pp. 108-114

15. CE Perkins, P Bhagwat, Highly dynamic destination-sequenced distancevector routing (DSDV) for mobile computers. Comput. Commun. Rev. 24(4), 234-244 (1994)

16. CC Chiang, HK Wu, W Liu, M Gerla, Routing in clustered multihop, mobile wireless networks, in Proc. of IEEE Singapore International Conference on Networks (SICON'97), ed. by (Singapore, Apr. 14-17, 1997), pp. 197-211

17. S Murthy, JJ Garcia-Luna-Aceves, A routing protocol for packet radio networks, in ACM/EEE MOBICOM, ed. by (Berkeley, CA, Nov. 1995), pp. 86-95

18. CE Perkins, Ad hoc on demand distance vector (AODV) routing. IETF Internet Draft (November 1998). [http://www.ietf.org/internet-drafts/draftietf-manet-aodv-02.txt]

19. DB Johnson, DA Maltz, Dynamic source routing in ad-hoc wireless networks, Mob. Comput. Chapter 5, 153-181 (1996)

20. ZJ Hass, The zone routing protocol (ZRP) for ad hoc networks, IETF, internet draft, draft-zone-routing-protocol-00.txt. (1997)

21. M Nickray, A Afzali-Kusha, R Jäntti, MEA: an energy efficient algorithm for dense sector-based wireless sensor networks. EURASIP J. Wirel. Commun. Netw. (2012)

22. PS Mundra, R Kumar, Energy efficient protocol for WSN. Int. J. Eng. Res. Appl. (IJERA) 2(3), 2912-2916 (2012)

23. A Dhawan, CT Vu, A Zelikovsky, Y Li, SK Prasad, Maximum lifetime of sensor networks with adjustable sensing range, in Seventh ACIS International Conference on Software Engineering, Artificial Intelligence, Networking, and Parallel/Distributed Computing (SNPD'06), ed. by (Las Vegas, 2006), pp. 285-289

24. J Wang, S Medidi, Energy efficient coverage with variable sensing radii in wireless sensor networks, in Third IEEE International Conference on Wireless and Mobile Computing, Networking and Communications, 2007, ed. by (WiMOB 2007, White Plains, NY, 8-10 Oct. 2007), p. 61

25. S Lin, J Zang, G Zhou, L Gu, T He, JA Stankovic, ATPC: adaptive transmission power control for wireless sensor networks, in ACM Conference on Embedded Networked Sensor Systems (ACM SenSys'06), ed. by (Boulder, Colorado, 2006), pp. 223-236

26. TS Rappaport, Wireless Communications: Principles and Practice (Prentice Hall, Englewood Cliffs, NJ, 2002

27. Wolfram Research, Mathematica. [http://www.worlfram.com/]

doi:10.1186/1687-1499-2012-343

Cite this article as: Cheng et al: MLPA-conservation mechanism in wireless sensor network environments. EURASIP Journal on Wireless Communications and Networking 2012 2012:343.

\section{Submit your manuscript to a SpringerOpen ${ }^{\circ}$ journal and benefit from:}

- Convenient online submission

- Rigorous peer review

- Immediate publication on acceptance

- Open access: articles freely available online

- High visibility within the field

- Retaining the copyright to your article

Submit your next manuscript at $\gg$ springeropen.com 